\title{
Evaluación de propagación asexual en dos especies de cactus: Corryocactus brevistylus K. Schum. y Oreocereus leucotrichus (Philippi) Wagenknecht, endémicos, pertenecientes al sector de precordillera de la XV región de Arica y Parinacota, Chile
}

\author{
Evaluation of asexual propagation by two cacti species: Corryocactus brevistylus K. \\ Schum., and Oreocereus leucotrichus (Philippi) Wagenknecht, native species in the \\ foothills of region XV, Arica and Parinacota, Chile.
}

Juan Trevizan Rispoli', Héctor Baltierra Chipana

\begin{abstract}
RESUMEN
Dos especies de la familia Cactaceae, Corryocactus brevistylus K. Schum. y Oreocereus leucotrichus (Philippi) Wagenknecht, pertenecientes al sector precordillerano de la XV Región de Arica y Parinacota, fueron propagadas asexualmente a partir de esquejes y tallos fragmentados (fracción con forma de cilindro y fracción con forma de triángulo), extraídos de individuos adultos y plantados sobre arena, para luego llevarlos a un sustrato definitivo, con el fin de establecer el mejor método de propagación asexual y así contribuir a la mantención de la diversidad biológica.

El estudio se dividió en tres etapas: cicatrización, enraizamiento y crecimiento. Durante el período de análisis se logró la formación de raíces, pero no fue suficiente como para poder evaluar la etapa de crecimiento. Por ello no se estableció un diseño experimental en esta etapa. La sobrevivencia en la etapa final del estudio arrojó que en la especie Corryocactus brevistylus, la propagación por esquejes logró el porcentaje más alto con un 76,7\% entre los tres tratamientos. Para Oreocereus leucotrichus el porcentaje más elevado también lo obtuvo la propagación por esquejes con un $30 \%$ de sobrevivencia entre los tres tratamientos.

Por tanto se concluye que la propagación vegetativa mediante esquejes es factible en Oreocereus leucotrichus.
\end{abstract}

Palabras clave: cactáceas, métodos de propagación, esquejes, Corryocactus brevistylus, Oreocereus leucotrichus

\begin{abstract}
Two species of the family Cactaceae, Corryocactus brevistylus K. Schum. and Oreocereus leucotrichus (Philippi) Wagenknecht, pertaining to the highland sector of XV the region of Arica and Parinacota, was propagated from shoots and of stems asexually fragmented (fraction with cylinder form, and faction with triangle form), extracted of adult and planted individuals on sand, soon to take them to a definitive substrate, with the purpose of establishing the best method of asexual propagation and contribute to the maintaining of the biological diversity.

The study was divided into three stages: healing, rooting and growth. During the analysis, it was possible root formation, but not sufficient it like being able to evaluate the growth stage. Therefore no experimental design was established at this stage.

The sobreexperience in the final stage of the study threw that in the Corryocactus brevistylus species, the propagation by shoots obtained the highest sobreexperience with a $76.7 \%$, between the three treatments. For Oreocereus leucotrichus the elevated percentage also scored propagation by shoots with a $30 \%$ of sobreexperience between the three treatments.

Therefore one concludes in this way, that the vegetative propagation by shoots is feasible in Oreocereus leucotrichus.
\end{abstract}

Key words: cactáceas, propagation methodes, shoots, Corryocactus brevistylus, Oreocereus leucotrichus

\section{Introducción y revisión bibliográfica}

Las cactáceas son plantas arborescentes o arbustivas, raras veces enredaderas, generalmente con hábito xerófito, de maderas blandas y cuerpo suculento que tiende a lignificarse con el tiempo. Este cuerpo puede estar constituido por segmentos, denominados "cladodios", de forma aplanada, cilíndrica o globosa (Hoffmann y Walter, 2004). Las cactáceas conforman cerca de 1.500 especies, todas ellas nativas del continente americano, cuya distribución abarca desde Canadá hasta la Patagonia, incluyendo las Antillas. Es en México donde se alberga la mayor riqueza con alrededor de 600 especies, lo que corresponde a cerca del 40\% de las familias (Reyes, 2009).

En Chile, las cactáceas corresponden a una de las familias de plantas superiores más ricas en endemismo: de las cerca de 143 especies, subespecies

\footnotetext{
1 Universidad de Tarapacá. Arica, Chile

Autor por correspondencia: jftrevizan@uta.cl; jftrevizan@gmail.com.
} 
y variedades descritas y clasificadas hasta ahora, más de 100 se hallan exclusivamente en nuestro país. Es decir, el $84 \%$ son endémicas - exclusivas de Chile - y el $14 \%$ del total se encuentran también en la flora de otros países limítrofes (Hoffmann y Walter, 2004).

Las cactáceas son plantas representativas del paisaje precordillerano chileno y desde tiempos muy remotos la madera de ellas se ha utilizado para hacer puertas, vigas, muebles, artesanías o como combustible. Esto ha provocado una disminución de las enormes poblaciones de cactus, llevando a muchas de ellas a una situación de alta vulnerabilidad (Arroyo y Quiroz, 2008).

Actualmente, la conservación in situ (en su hábitat) constituye una herramienta importante en la preservación de la diversidad de este grupo (PNUMA, 2011; CONABIO, 1998).

Por ello es importante desarrollar estrategias de conservación para la protección de dichas especies, así como estudiar y comprender su biología para ayudar a restablecer sus poblaciones naturales y asegurar su preservación (Gliessman, 2002).

Una alternativa para la solución de esta problemática es la utilización de técnicas sencillas y factibles para la multiplicación de las cactáceas, lo que involucra la evaluación de nuevos métodos de propagación, además de sustratos que proporcionen los nutrientes adecuados para el óptimo crecimiento de las plantas (Reyes, 2009).

En consecuencia, se pretende establecer una metodología para la propagación de cactáceas seleccionadas, que permita disponer de material para su reproducción y disminuir la presión sobre estas poblaciones vegetales.

Dada la importancia de Corryocactus brevistylus K. Schum. y Oreocereus leucotrichus (Philippi) Wagenknecht para la conservación de la diversidad de las poblaciones vegetales en el sector precordillerano del norte de Chile y la poca información existente respecto a sus estados fenológicos y medios de propagación, se hace necesario aportar mayores antecedentes que permitan conocer su comportamiento y con esto establecer medidas más racionales para su preservación. El objetivo es evaluar la supervivencia de plantas durante el proceso de enraizamiento con diferentes tipos de propagación.

La familia Cactaceae es de origen americano y posee una distribución desde Canadá a Tierra del Fuego, con mayor diversidad en México. Se estima que existen unos 86 géneros y alrededor de 2.000 especies. En Chile, la flora de las cactáceas está representada por unas 160 especies, de las cuales 145 son endémicas, la mayoría amenazadas (Hoffmann y Walter, 2004).

Debido a la degradación de los hábitats naturales y a la sobrecolecta (principales causas de la reducción de las poblaciones silvestres de cactáceas), muchas han devenido en especies en peligro de extinción o incluso, extintas. Por estoes importante el desarrollode estudios multidisciplinarios encaminados a conservar y rescatar las plantas suculentas raras y amenazadas, y a proteger las que aún son relativamente abundantes (Benson, 1977).

Poco se sabe de la ecología, fisiología, reproducción y tendencia evolutiva de las poblaciones naturales de cactus, y menos aún de una evaluación cuantitativa de ellas. Se presums que tienen, por lo general, una difícil regeneración natural y un crecimiento extremadamente lento (Soberón, 1998).

Son objeto de una fuerte predación, tanto por agentes de su hábitat como por el hombre, quien, por desconocimiento y falta de respeto, o como consecuencia del desarrollo de la agricultura y la minería o del incremento de las ciudades y vías de comunicación, ha destruido los ecosistemas (Dajoz, 2001).

Las especies chilenas, aunque estén protegidas internacionalmente por la legislación de CITES, han sido sometidas a una constante explotación extractiva y a una corta indiscriminada para su uso como combustible y como forraje, o, simplemente, por falta de conocimiento acerca de su importancia ecológica (Benoit, 1989). Los cambios en el clima y la desertificación son factores de riesgo y preocupación que también hay que mencionar, especialmente para las especies nortinas.

En efecto, representan un grupo importante de plantas típicas de las zonas áridas y semiáridas de Chile, donde muy pocos vegetales crecen, dando al paisaje una fisonomía especial, de gran valor estético, científico y turístico (Hoffmann y Walter, 2004).

La biología de la conservación como ciencia surge debido a que ninguna de las ciencias actuales por sí sola podía responder a la creciente crisis que afronta la diversidad biológica (Primak, 1993), y plantea que para mantener la biodiversidad pueden utilizarse las técnicas de conservación in situ, ex situ y la integrada (Mc Neely et al., 1990).

Las cactáceas poseen una serie de adaptaciones morfofisiológicas adquiridas en respuesta a las presiones del medio árido. La adaptación fisiológica más evidente es su capacidad de almacenar y conservar agua en sus tejidos (Oudshoorn, 1978). 
Entre las adaptaciones morfológicas más evidentes se encuentran la reducción de la superficie transpiratoria al adquirir formas globulares y/o esféricas; la modificación de las hojas en espinas que impiden una transpiración excesiva, condensan el agua atmosférica y protegen contra la herbivoría; el desarrollo de epidermis y cutícula gruesa y casi sin poros, muchas veces recubierta de una abultada capa de cera; la disposición hundida de los estomas que determina la formación de espacios aéreos que se saturan de vapor de agua y permiten que la transpiración disminuya; y las adaptaciones que posibilitan la rápida absorción del agua como la gran longitud y ramificación de sus raíces (Taiz y Zeiger, 2006).

Numerosas especies de cactus, al plantarse trozos de su cuerpo, producen raíces con facilidad. Del mismo modo, si a plantas adultas se les cortan drásticamente las raíces, estas se regeneran rápidamente cuando las condiciones del medio son apropiadas (Hoffmann y Walter, 2004).

En ciertos especímenes en cultivo, es corriente que las raíces se vean infectadas de hongos $u$ otros microorganismos que provocan la pudrición o la aparición de diversas enfermedades. Es por esto que, antes de ser plantadas, las raíces deben ser cuidadosamente podadas, secadas al aire y desinfectadas. Si alguna especie produce raíces con dificultad, es posible injertarlas sobre un pie previamente enraizado, que les procurará el agua y los nutrientes necesarios (Hoffmann y Walter, 2004).

Los tallos de las cactáceas son de consistencia suculenta y, por lo general, de color verde, sobre todo en su estado juvenil, a menudo lignificándose y recubriéndose de una voluminosa corteza en la edad avanzada (Hoffmann y Walter, 2004).

Con frecuencia el tallo se encuentra articulado en secciones denominadas "cladodios" o "filocladios". En la mayoría de los géneros, los tallos están surcados de hendiduras longitudinales que producen crestas sobresalientes, llamadas "costillas". Las costillas hacen muy resistentes los tallos a las fuerzas de flexión, y a menudo presentan protuberancias suculentas llamadas "mamilas" o "tubérculos", que pueden variar mucho en su configuración, dando a los cactus su aspecto peculiar. Esta superficie irregular permite a la planta encogerse en los períodos de sequía y soportar pérdidas de volumen, que en algunas oportunidades alcanzan más de un tercio de su peso (Hoffmann y Walter, 2004).

Existen dos métodos para la propagación de cactáceas: los métodos de propagación biotecnológica (a través del cultivo de tejidos) y los de propagación convencional (a través de la propagación por semilla y propagación vegetativa: hijuelos, esquejes o injertos). (Rubluo et al., 1996; Rojas y Vásquez, 2000). Sin embargo, la propagación convencional en cactáceas representa una alternativa para los países carentes de tecnología y recursos económicos que permitan el aprovechamiento comercial de sus recursos naturales (Reyes, 2009).

La propagación por semillas es la más importante debido a que la mayoría de las cactáceas y suculentas produce gran cantidad de semillas y permite la obtención de miles de plantas con variación genética, factor primordial para un programa de restauración ecológica (Álvarez y Montaña, 1997).

Es un proceso difícil de darse en su medio natural, debido a los problemas de germinación que presentan las semillas producto de las condiciones ambientales adversas a las cuales se encuentran sometidas (Maiti et al., 2002). En la mayoría de ellas se necesitan tres factores: agua, temperatura y luz, para lograr que germinen (Vásquez et al., 1995).

Propagar plantas a través de semillas resulta más lento que hacerlo por reproducción vegetativa, pero es la forma más sencilla y económica de obtener una colección variada. Por otra parte, las semillas ayudan a crear reservas de plantas que se encuentran en peligro de extinción (Toogood, 2002).

Los vástagos o hijuelos son brotes que proliferan en algunas cactáceas globosas. Esta técnica de multiplicación es fácil, ya que se trata de desprender los brotes que emergen alrededor de la planta madre. Una vez separados se dejan cicatrizar entre 10 y 15 días en un sitio seco y ventilado, y posteriormente se plantan. La ventaja de este método es la rápida obtención de plantas adultas y la desventaja es la carencia de recombinaciones genéticas, importantes en la conservación de especies (López, 2006).

Dividir los cactus y otras plantas suculentas es una tarea relativamente sencilla y supone una manera única de propagación de híbridos, formas selectas y plantas variegadas que no suelen reproducirse fielmente a partir de semillas (Harman y Kesters`s, 2002).

Algunos cactus y otras plantas suculentas no florecen bien y no siempre es fácil conseguir semillas, por lo que los esquejes se convierten en la opción más viable para su propagación. Los esquejes de plantas suculentas tienen la ventaja de que, gracias a su tejido carnoso, conservan nutrientes y agua que utilizarán hasta el momento que se establezcan en maceta (Harman y Kester's, 2002). 
El método asexual es el más fácil para propagar cactáceas y consiste en fragmentar un tallo en varios trozos, que deben dejarse cicatrizar en un lugar seco y ventilado (López, 2006).

El mejor momento del año para tomar los esquejes de la mayoría de los cactus es a finales de la primavera, sobre todo en las zonas de clima frío, ya que se inicia la época de calor, el ambiente está seco y las plantas comienzan su crecimiento más intenso.

Otra alternativa es proporcionarles a los esquejes las condiciones adecuadas de temperatura, humedad y luz para su enraizamiento (Toogood, 2002). La mayor parte de los cactus columnares se pueden propagar mediante esquejes de tallo.

Los esquejes deben hacerse de plantas adultas o en etapa de producción, ya desarrolladas, que tengan varios años (Rodríguez, 1997) y las plantas madre deben ser vigorosas, no presentar daños de sol, plagas y/o enfermedades (Livera, 1991).

\section{Materiales y métodos}

Corryocactus brevistylus (Schumann) Britton \& Rose. El cactus $C$. brevistylus se conoce con el nombre común de "guacalla" o "quisco de flores amarillas". Es una especie no endémica, clasificada como vulnerable en su hábitat natural por el Libro Rojo de laFlora Terrestre Chilena (Benoit, 1989). Pero según antecedentes de Pinto y Kirberg (2009), esta especie es una de las más abundantes de la precordillera, aunque su aspecto general es bastante deplorable. Se encuentra desde Arequipa, en el Perú, hasta Mamiña, al interior de Iquique, en la precordillera andina (2.400 a $3.500 \mathrm{msnm}$ ) (Hoffmann y Walter, 2004). Últimos registros indican que esta especie se extiende hasta el sur de la Quebrada de Choja (21 $11^{\circ}$ 'sur) al sur de Huatacondo, y existe un tramo con ausencia de cactus que corresponde a los arenales altos de Pica (Pinto y Kirberg, 2009).

De acuerdo con Hoffmann y Walter (2004), presenta un cuerpo arbustivo o a menudo arborescente, de $1,5 \mathrm{a} 5 \mathrm{~m}$ de altura, con 6 a 9 costillas, triangulares en corte transversal, cuya epidermis es de una coloración verdosa. Llega a medir hasta $50 \mathrm{~cm}$ de diámetro. Espinas derechas, amarillas a rojizas, aciculares, no bien definidas en centrales y marginales. Presenta aréolas redondeadas. Respecto a las flores laterales, son diurnas, sin perfume, y miden entre 8 y $11 \mathrm{~cm}$.

Los frutos son grandes, redondos, verde oliva, de carne ácida y jugosa, que contiene numerosas semillas marrón (Hoffmann y Walter, 2004). El fruto se come en ayunas en las mañanas con sal o azúcar. Además se usa como medicina para afecciones hepáticas y diabetes, como regulador del colesterol y laxante, para tratar alopecia, caspa y seborrea (Pinto y Kirberg, 2009).

Reino: Plantae; División: Magnoliophyta; Orden: Caryophyllales; Familia: Cactaceae; Género: Corryocactus Britton \& Rose; Especie: C. brevistylus; Nombre binomial: Corryocactus brevistylus (K. Schum. ex Vaupel) Britton \& Rose; Sinonimia: Cereus brevistylus K. Schum., C. brevistylus subesp. Brevistylus, C. brevistylus subesp. Puquiensis, C. brevispinus, $C$. heteracanthus, $C$. krausii, $C$. pachycladus, C. ayacuchoensis.

Oreocereus leucotrichus (Philippi) Wagenknecht.

$\mathrm{El}$ cactus $O$. leucotrichus se conoce con el nombre común de "viejito" o "chastudo". Es una especie no endémica, clasificada como sin problemas de conservación en su hábitat natural por el libro Cactáceas en la Flora Silvestre de Chile (Hoffmann, 1989). Según observaciones de Pinto y Kirberg (2009), debe catalogarse como vulnerable ya que a pesar de su amplia distribución, las poblaciones con gran densidad de plantas son muy localizadas. La abundancia y el vigor de las plantas también varían con la altitud, encontrándose plantas más débiles y menos abundantes a menores altitudes (Pinto y Kirberg, 2009).

Se encuentra desde cerros de la precordillera nortina, desde Vilama (cerca de Calama) hasta Chiral (Arequipa), en el Perú. También en regiones de Arica y Parinacota, Tarapacá y Antofagasta (Hoffmann y Walter, 2004). Crece en la alta precordillera entre 3.000 y 3.800 m (Pinto y Kirberg, 2009).

Esta especie presenta gran variabilidad morfológica, de acuerdo a la altitud y condiciones climáticas puntuales del lugar donde se hallan las plantas. En niveles altitudinales superiores se encuentran plantas de tallos gruesos y muy peludos. En niveles altitudinales más bajos se hallan plantas de tallos más delgados, con menor número de costillas y más profundas, aréolas con menos pelos, con mayor número de espinas y más gruesas y de flores más pequeñas. En altitudes intermedias es posible encontrar ambas morfologías.

De acuerdo con Hoffmann y Walter (2004), presenta un cuerpo arbustivo, con ramificaciones desde la base, de 1 a $2 \mathrm{~m}$ de altura, y 6 a $12 \mathrm{~cm}$ de diámetro. Con 12 a 15 costillas, aréolas con abundantes pelos sedosos, blancos, rojizos o negros. Espinas amarillas, marrones o anaranjadas. 
Las flores cerca del ápice, rojas con visos violetas. Los frutos redondos, y amarillentos, de 4 a $6 \mathrm{~cm}$ de diámetro, como se observa en la Figura 1 (Hoffmann y Walter, 2004).

Reino: Plantae; División: Magnoliophyta; Orden: Caryophyllales; Familia: Cactaceae; Género: Oreocereus (A. Berger) Riccob; Especie: $O$. leucotricha;_Nombre binomial: Oreocereus leucotrichus (Philippi) Wagenknecht; Sinonimia: Arequipa leucotricha, Borzicactus leucotrichus, Echinocactus leucotrichus, Borzicactus hendriksenianus, Oreocereus hendriksenianus, Oreocereus ritteri, Oreocereus varicolor, Morawetzia varicolor.

La presente investigación se llevó a cabo durante los años 2011 y 2012 en la localidad de Chapiquiña, a una altitud entre los 3.317 y $4.300 \mathrm{msnm}$, comuna de Putre, Región de Arica y Parinacota, Chile.

En la estepa prealtiplánica es característico el Corryocactus brevistylus en el límite inferior y Oreocereus leucotrichus en el límite superior (Pinto y Kirberg, 2009 y Arroyo, 1997).

Se elaboró un protocolo simple de recolección de material vegetal, haciendo alusión a la propagación de la gran familia de las cactáceas en la literatura especializada (Johnson y Emino, 1979; Reyes, 2009). Para realizar la propagación de cactus se utilizó el método asexual: método de propagación por fragmentación y por esqueje, el cual consiste en fragmentar un tallo en varios trozos, que deben dejarse cicatrizar en un lugar seco y ventilado (Marsden, 1960).

Se colectaron brazos de 1,5 m de altura en promedio. Estos brazos se cortaban de forma tangencial a nivel de la base del tallo de los cactus, con un serrucho previamente desinfectado en alcohol. Después se espolvoreaba con un fungicida alrededor de la herida realizada a la planta madre, para evitar presencia de hongos.

Una vez recolectado el material vegetal se realizaron cortes fraccionados con forma de triángulos, de 12 y $15 \mathrm{~cm}$ de largo para ambas especies y se colectaron esquejes de Oreocereus leucotrichus y Corryocactus brevistylus, con un tamaño que variaba entre 12 y $23 \mathrm{~cm}$. Además de esquejes, se colectaron brazos completos de ambas especies para realizar cortes fragmentados con forma de cilindro de 10 a 17 $\mathrm{cm}$, aproximadamente. Todos fueron asperjados con una solución de Captan W80, con el fin de prevenir enfermedades fungosas para las dos especies de cactáceas en estudio.

El procedimiento expuesto a continuación corresponde a la segmentación de los tallos de los cactus columnares en ambas especies (fracciones en forma de cilindro y triángulo).

Se comienza la limpieza con alcohol de $95 \%$ de pureza de los materiales que se utilizaron para realizar los cortes, como serrucho, machete y mesa de apoyo. Los grandes brazos de los cactus se cortaron transversalmente, formando así los fragmentos con forma de cilindro, como se observa en la Figura 1 c y d. Los trozos cortados medían de 10 a $17 \mathrm{~cm}$ para obtener un mínimo de 4 aréolas por costillas en ambas especies. De ellos, al menos dos debían quedar enterrados en el sustrato.

Para realizar las fracciones con forma de triángulo se cortaron en trozos de cactus columnar de 12 a $15 \mathrm{~cm}$ de largo, para después segmentarlos en 4 trozos más pequeños, como se puede observar en la Figura $1 \mathrm{a}$ y $\mathrm{b}$.

En los esquejes de cactus columnares se cortaron las irregularidades realizadas al momento de colectar el material vegetal. La longitud de los esquejes variaba entre 12 y $23 \mathrm{~cm}$ (Figura 1 e y f).

Con la finalidad de prevenir problemas con enfermedades fúngicas, se deben desinfectar todos los fragmentos y esquejes en una solución de hipoclorito de sodio con agua corriente $(0,11 / 501)$ durante 5 segundos. Se dejaron escurrir y posteriormente fueron tratados con una solución de Captan W80 (10g/ 250 $\mathrm{ml}$ de agua). Tanto las fracciones como los esquejes se ubicaron sobre mesones de madera al interior de un galpón cerrado pero con adecuada ventilación. Aquí permanecieron durante 15 a 30 días para permitir la cicatrización de la zona del corte, con el fin de evitar la presencia de hongos sobre la herida.

Se pesaron trozos y esquejes de ambos cactus en estudio para determinar la pérdida de peso en el tiempo. Se registró la deshidratación de los tres tratamientos (esqueje, cilindro y triángulo) con una balanza analítica. El período de registro del peso se realizó en los meses de julio, agosto, septiembre y octubre. Estos trozos de cactus no fueron los que se utilizaron en el período de enraizamiento, ya que no debían moverse de forma permanente para dar paso al enraizamiento sobre el sustrato transitorio (arena). El fin de determinar la pérdida de peso fue establecer una relación entre la deshidratación y la supervivencia en los trozos de cactus.

En la Figura 2 se observa un resumen de la forma de propagación que se realizó en las dos especies de cactus, Corryocactus brevistylus y Oreocereus leucotrichus. Al obtener los trozos de cactus con forma de cilindro, estos se cortan nuevamente en cuatro partes, formando así 


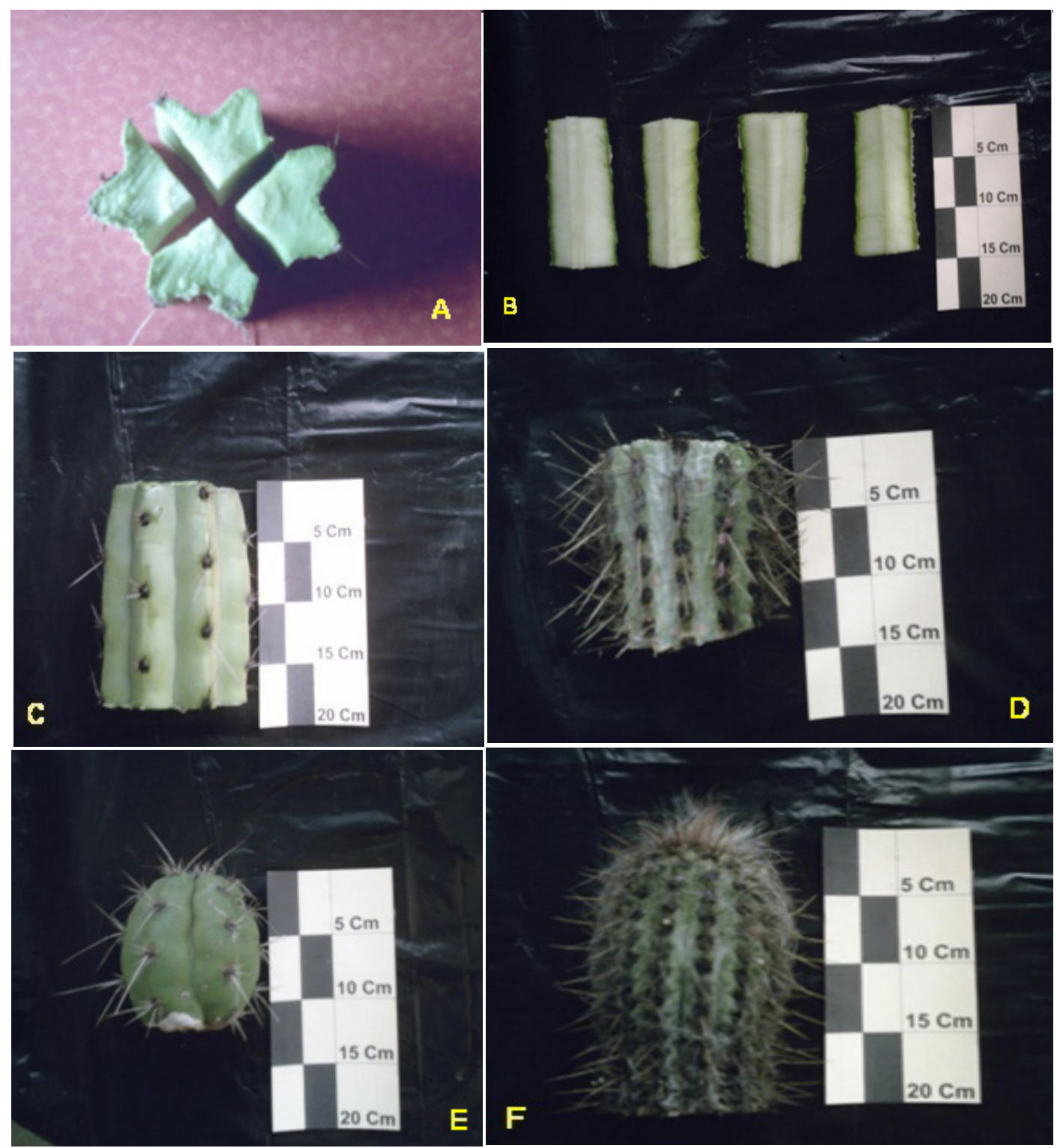

Figura 1. Métodos de Propagación en Cactáceas: Propagación por fragmentación (A, B, C y D) y propagación por esqueje (E y F). Fracciones con forma de triángulo (A y B), fracciones con forma de cilindro (C y D), y esquejes (E y F). Especie Corryocactus brevistylus (A, C y E) y la especie Oreocereus leucotrichus (B, D y F). Fuente: Elaboración propia. 


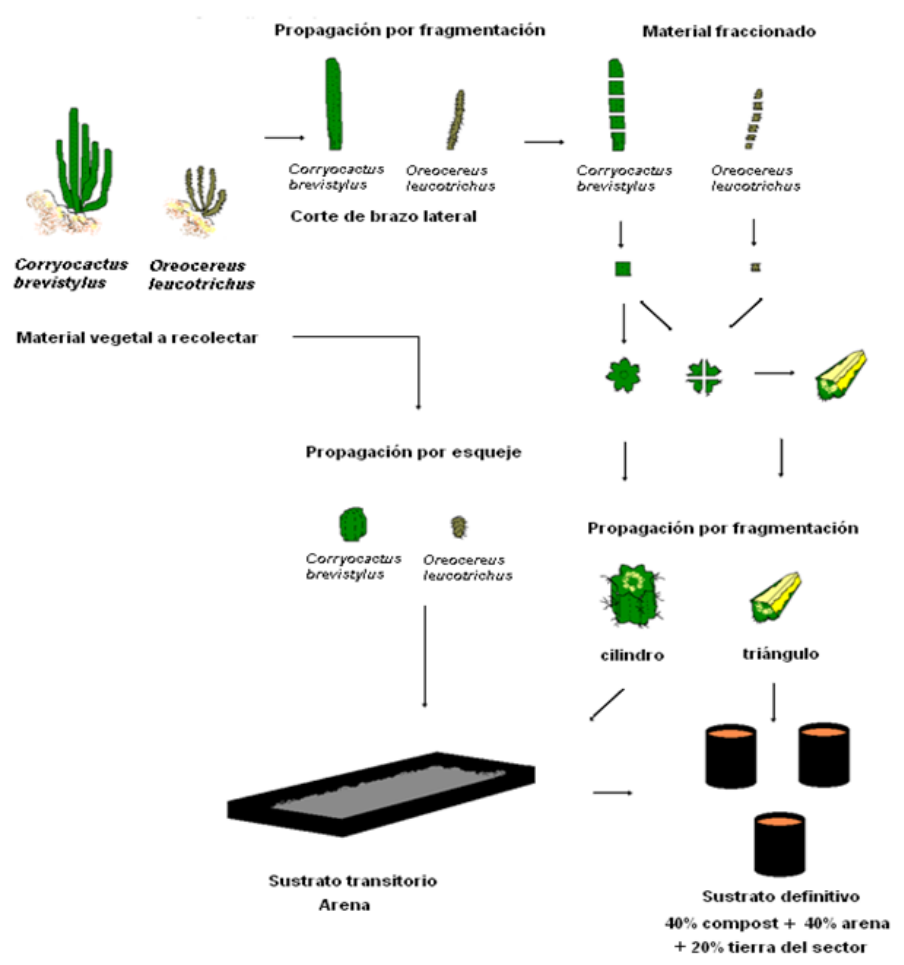

Figura 2. Ejemplo de Propagación de cactus en las especies Corryocactus brevistylus y Oreocereus leucotrichus. Fuente: Elaboración propia.

trozos de cactus con forma de triángulo. La propagación por esqueje fue directa ya que se obtuvo de plantas madre con una longitud de $15 \mathrm{a} 20 \mathrm{~cm}$. Los esquejes y los trozos con forma de cilindro fueron plantados sobre un sustrato transitorio en platabanda, mientras que los trozos con forma de triángulo se plantaron sobre un sustrato definitivo en maceta.

Después del período de secado se realizó la plantación de las fracciones. Los segmentos (triángulo) se plantaron sobre sustrato definitivo (arena + compost + tierra del sector) en bolsas de polietileno de 15 x $30 \mathrm{~cm}$. Los segmentos (cilindro) y los esquejes se plantaron sobre sustrato transitorio (arena). En la plantación del material vegetal se contabilizó la supervivencia de los trozos de cactus vivos, tomando como criterio visual la presencia de hongos así como la pérdida de tejido vegetal por deshidratación.

Una vez plantados sobre sustrato se les proporcionó riego mediante una regadera. El riego se realizaba cada 16 días sobre el sustrato transitorio (arena) de forma superficial.

El ensayo estuvo compuesto de tres etapas: la primera fue el secado o cicatrización, seguido del enraizamiento y por último el crecimiento y desarrollo vegetativo del trozo enraizado. Durante los 30 días del período de secado, se registró la deshidratación de los tres tratamientos (esqueje, cilindro y triángulo) en ambas especies, con una balanza analítica, además de observar la presencia de hongos y variación de la coloración en la superficie del cactus.

En la etapa de enraizamiento se observó la aparición de raíces. Una vez alcanzada una longitud mínima, se dio inicio a la tercera etapa, con la plantación de los fragmentos y esquejes en el sustrato definitivo. En esta etapa se observaría el crecimiento de nuevos brotes o algún cambio en la morfología de estos.

El estudio corresponde a un ensayo realizado en un vivero bajo un diseño experimental completamente 
aleatorio. Se hicieron 3 tratamientos (esqueje, cilindro y triángulo) y 3 repeticiones, dando un total de 9 unidades experimentales para cada especie. Cada unidad experimental contó con 10 trozos o esquejes, dispuestos en una platabanda de 1,2 x $5 \mathrm{~m}$, para cada una de las especies.

Para el análisis de los factores en estudio se utilizó la técnica del análisis de varianza, aplicando la prueba $\mathrm{F}$ a un nivel de significación de 0,05 . Para la comparación de medias de cada factor se usó la prueba de significación de Tukey a una probabilidad $\alpha=0,05$.

\section{Resultados}

Para la especie Corryocactus brevistylus existe un $100 \%$ de mortalidad de los individuos que corresponden a los trozos con forma de triángulo y un 73,34\% para los trozos con forma de cilindro. En la especie Oreocereus leucotrichus hay un $100 \%$ de mortalidad que corresponde a los trozos con forma de triángulo y cilindro.

En la Figura 4 se muestra la deshidratación en los 3 tratamientos (esqueje, cilindro y triángulo) de la especie $C$. brevistylus. Se observa que T3 presenta el mayor porcentaje de deshidratación, seguido por T2 y después T1. El T3 tuvo 95,05\% de deshidratación con una desviación estándar de 1,02\%, T2 un 32,05\% con una desviación estándar de $0,97 \%$ y T1 obtuvo una deshidratación de $14,15 \%$ con una desviación estándar de 1,96\%, demostrando así que entre los tratamientos existen diferencias estadísticas significativas mediante la prueba de Tukey $(\mathrm{P}<0,05)$. El coeficiente de variación de 2,84\% es aceptable para las condiciones del experimento.

En la Figura 5 se observa el porcentaje de supervivencia en los 3 tratamientos (esqueje, cilindro y triángulo) de la especie C. brevistylus. Se puede apreciar que $\mathrm{T} 1$ presenta el mayor porcentaje de supervivencia, seguido por T2 y después T3, (anexos 10 y 11). El T1 tuvo 76,7\% de supervivencia con una desviación estándar de 15,3\%, T2 un 26,7\% con una desviación estándar de $15,28 \%$ y en T3 no hubo supervivencia, demostrando así que entre los tratamientos existen diferencias estadísticas significativas mediante la prueba de Tukey $(\mathrm{P}<0,05)$. El coeficiente de variación de $28,12 \%$ es aceptable para las condiciones del experimento.

Para determinar la pérdida de peso porcentual del material vegetal durante la etapa de secado o cicatrización se registraron cada 13 días durante 4 meses los pesos de los 3 tratamientos en ambas especies de cactus. Los registros fueron en julio, agosto, septiembre y octubre del 2011.

En la Figura 6 se observa la deshidratación en los 3 tratamientos (esqueje, cilindro y triángulo) de la especie Oreocereus leucotrichus. Se aprecia que T3 presenta el mayor porcentaje de deshidratación, seguido por T2 y después T1 (anexos 14 y 15). El T3 tuvo 91,3\% de deshidratación con una desviación estándar de 2,35\%, T2 un 41,74\% con una desviación estándar de 3,24\% y T1 obtuvo una deshidratación de $18,53 \%$ con una desviación estándar de $0,81 \%$, demostrando así que entre los tratamientos existen diferencias estadísticas significativas mediante la prueba de Tukey $(\mathrm{P}<0,05)$. El coeficiente de variación de 2,49\% es aceptable para las condiciones del experimento.

En la Figura 7 se muestra el porcentaje de supervivencia en los 3 tratamientos (esqueje, cilindro y triángulo) de la especie Oreocereus leucotrichus. Se puede apreciar que $\mathrm{T} 1$ fue el único que sobrevivió en la etapa de enraizamiento con $30 \%$ de supervivencia.

\section{Conclusiones}

De acuerdo a los análisis estadísticos realizados, la técnica de triángulo en ambas especies de cactus (C. brevistylus y $O$. leucotrichus) fue la que presentó una mayor deshidratación en el tiempo. Asimismo, su evolución de deshidratación demuestra que en una primera instancia estos segmentos sufren una pérdida de agua abrupta en ambos casos en contraste con los otros tratamientos. Esto se debería a la pérdida parcial de la cutícula, la cual es una barrera que dificulta su evaporación. Según Pérez (1994) la cutícula es una capa impermeable que recubre la epidermis de los órganos herbáceos expuestos a la atmósfera y que reducen en gran medida las pérdidas por evaporación.

Asimismo, la supervivencia de los segmentos de cactus en las dos especies en estudio sometidos a la técnica de corte en triángulo (T3) evidencia una drástica disminución, llegando al total de la mortalidad de los segmentos en la etapa de enraizamiento. Esta mortalidad se demuestra también en la técnica de corte en cilindro en la especie $O$. leucotrichus con un porcentaje de supervivencia del $0 \%$. Es así como la epidermis de este tipo de plantas juega un rol fundamental en la deshidratación de los segmentos, afectando severamente su capacidad de retención de agua (Farré, 2005).

En contraste con el triángulo está la técnica del esqueje, que sufrió una deshidratación mínima o moderada con solo un $14,15 \%$ en la especie $C$. 


\begin{tabular}{|c|c|c|c|}
\hline & T1 & $\mathrm{T} 2$ & T3 \\
\hline R 1 & 10 & 10 & 10 \\
\hline R 2 & 10 & 10 & 10 \\
\hline R 3 & 10 & 10 & 10 \\
\hline
\end{tabular}

Corryocactus brevistylus

\begin{tabular}{l|c|c|c|}
\multicolumn{1}{c}{} & \multicolumn{1}{c}{ T1 } & \multicolumn{1}{c}{ T2 } & \multicolumn{1}{c}{ T3 } \\
\cline { 2 - 4 } R1 & 10 & 10 & 10 \\
\cline { 2 - 4 } R2 & 10 & 10 & 10 \\
\cline { 2 - 4 } R3 & 10 & 10 & 10 \\
\cline { 2 - 4 } & &
\end{tabular}

Oreocereus leucotrichus

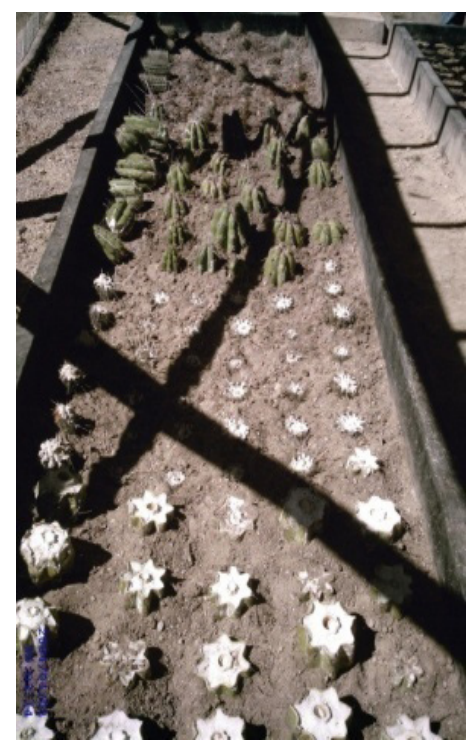

Figura 3 Plantación del material vegetal de ambas especies y esquema del ensayo, Corryocactus brevistylus y Oreocereus leucotrichus. Fuente: Elaboración propia.

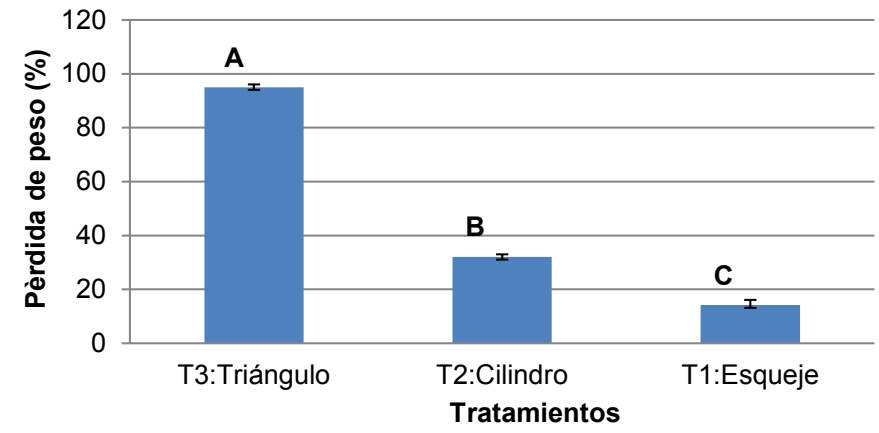

Figura 4. Deshidratación porcentual final de los 3 tratamientos en la especie Corryocactus brevistylus. Letras diferentes indican diferencias estadísticas significativas, de acuerdo a la prueba de Tukey $(\mathrm{P}<0,05)$. 


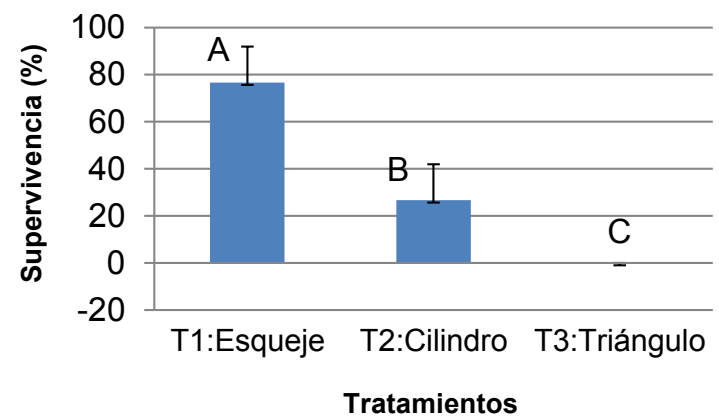

Figura 5. Supervivencia porcentual final de los 3 tratamientos en la especie Corryocactus brevistylus. Letras diferentes indican diferencias estadísticas significativas, de acuerdo a la prueba de Tukey $(\mathrm{P}<0,05)$.

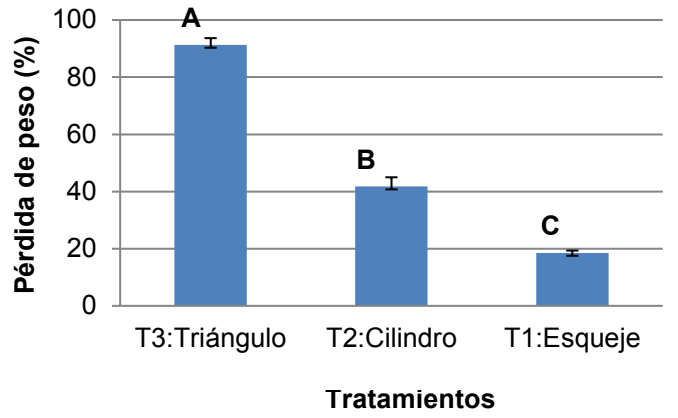

Figura 6. Deshidratación porcentual final de los tres tratamientos en la especie Oreocereus leucotrichus. Letras diferentes indican diferencias estadísticas significativas, de acuerdo a la prueba de Tukey $(\mathrm{P}<0,05)$.

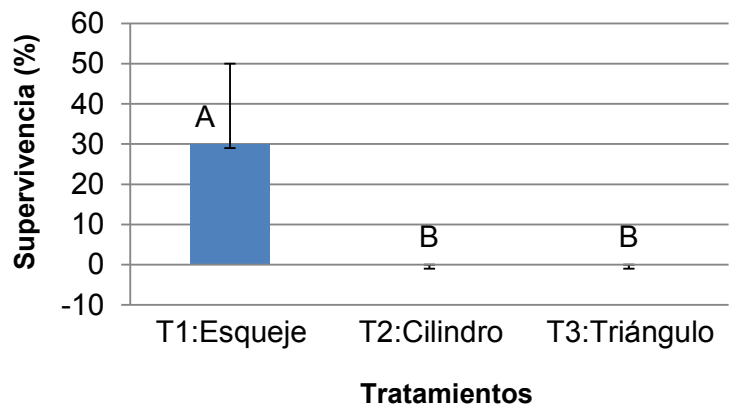

Figura 7. Supervivencia porcentual final de los tres tratamientos en la especie Oreocereus leucotrichus. Letras diferentes indican diferencias estadísticas significativas, de acuerdo a la prueba de Tukey $(\mathrm{P}<0,05)$. 
brevistylus y un $18,53 \%$ en $O$. leucotrichus, debido a su pequeña herida en la base, a diferencia de los otros tratamientos (Farré, 2005).

Con el fin de generar mayor información se recomienda realizar más estudios que optimicen los métodos convencionales de propagación para acortar los tiempos en la obtención de cactus e incrementar el número de individuos.

Debido a que no hay literatura ni ensayos para estas especies, por lo cual se deben descartar algunas técnicas realizadas, es importante probar nuevas estrategias de reproducción convencional, fechas de colectas del material vegetal y otro tipo de sustratos.

Se recomienda también determinar, mediante investigaciones, métodos convencionales con semillas, ya que estos son los más importantes porque permiten la obtención de plantas con variación genética o estudios de propagación en condiciones controladas.

\section{Literatura citada}

Álvarez A.; Montaña, C.

1997. Germinación y supervivencia de cinco especies de cactáceas del Valle de Tehuacán: implicaciones para su conservación. Acta de Botánica Mexicana, 40: 35-58.

Arroyo-Quiroz, I., Mueller, E.; Ortiz, B., Padua, S.M.; Pérez Gil-Salcido, R.; Rodríguez, C.M.

2008. Intercambio de experiencias sobre conflictos entre humanos y vida silvestre en Latinoamérica. UICN-Congreso Mundial de Conservación. Barcelona, España.

Arroyo, M.T.K.; Squeo, F.; Veit, H.; Cavieres, L., León, P.; Belmonte, E. 1997. Flora and Vegetation of Northern Chilean Andes. En: Actas del II Simposio Internacional de Estudios Altiplánicos. El Altiplano, ciencia y conciencia en los Andes, Universidad de Chile. Santiago, Chile. pp. 167-178.

Benoit, I.

1989. Red Book of Chilean Terrestrial Flora (Part One). Corporación Nacional Forestal (CONAF). Santiago, Chile. 151 p.

Benson, L.

1977. Preservation of Cacti and Mangement of Ecosystem. En: Prance, G.T., Elías, T.S. (Eds.). Extinction is forever. New York Botanical Garden. N.Y., U.S. pp. 283-300..

CONABIO.

1998. La Diversidad Biológica de México: Estudio de País, Comisión Nacional para el Conocimiento y Uso de la Biodiversidad. México. D.F., México. 293 p.

Dajoz, R.

2001. Tratado de Ecología. España, Editorial Mundi-Prensa. $480 \mathrm{p}$.

Farré, A C.

2005. El Gran Libro de los Cactus y otras Plantas Crasas. Ed. Cecchi. Barcelona, España. 193 p.

Gliessman, S.

2002. Agroecología: Procesos Ecológicos en la Agricultura Sostenible. LITOCAT. San José, Costa Rica. 359 p.

Gómez, A.

2000. Enciclopedia Ilustrada de los Cactus y otras Suculentas. Editorial Floramedia. España. 226 p.

Harman, L.; Kester's, M.

2002. Plant Propagation. Principles and Practices. Edition 7th. Ed. Hudson T. Prentice Hall New Jersey, U.S. 928 p.

Hoffmann, A.; Walter, H.

2004. Cactáceas en la flora silvestre de Chile. $2^{\circ}$ edición. Ediciones Fundación Claudio Gay. Santiago, Chile. $304 \mathrm{p}$.
Livera M., M.

1991. Retos y perspectivas del fitomejoramiento: Algunos aspectos del ambiente físico en relación a la producción y productividad de los cultivos. Rev. Fitotecnia Mexicana, 14: 23-29.

Marsden, C.

1960. Cultivo de Cactos. Barcelona, España. Garriga. 207 p.

McNeely,J.A.;Miller,K.R.; Reid, W.V.; MittermeierI,R. A.; Werner,T.B. 1990. Conserving the World Biological Diversity. IUCN, WRI, CI, WWF-US, the World Bank. Gland, Seize. 193 p. Oudshoorn, W.

1978. 126 Cactos y Plantas Crasas en Color. Editorial Instituto Parramón. España. 112 p.

Pérez, F., Martínez-Laborde, J. 1994. Introducción a la Fisiología Vegetal. Ed. Mundi-Prensa. Madrid, España. 218 p.

Pinto, R.; Kirberg, A. 2009. Cactus del Extremo Norte de Chile. Ed. Autoedición. Santiago, Chile. 246 p.

PNUMA

2011. Desertificación y degradación de suelos. Programa de las Naciones Unidas para el Medio Ambiente. Disponible en: http://www.pnuma.org/nuestra_mision/index. php?menusup=1\&menuinf=1PALC.): Consultado: $8 / \mathrm{feb} / 2011$.

Primack, R.B.

1993. Essential of Conservation Biology. Sinauer Associates, Inc. USA.En: Arango, N.; Chávez,M.E. 2003. Aportes conceptuales a la discusión de vulnerabilidad de áreas protegidas. pp. 8.

Reyes, J.

2009. Conservación y Restauración de Cactáceas y otras Plantas Suculentas Mexicanas. Comisión Nacional Forestal (CONAFOR). Jalisco, México. 101 p.

Rodríguez, C. A.

1997. Guía Técnica para la Producción de Plantas de Pitaya en Vivero. Secretaría de Desarrollo Social- Universidad Autónoma de Chapingo. México. 53 p.

Rojas M., A.; Vásquez Y., C.

2000. Cactus seed germination: a review. Journal of Arid Environments, 44: 85-104.

Rubluo, A., Reyes, J.; Rodríguez., G, B.; Pimienta, B, E.; Brunner, I. 1996. Métodos de Propagación Biotecnológicos y Convencionales en Cactáceas para Zonas Áridas. En: FAO. Técnicas convencionales y Biotecnológicos para la propagación de plantas de zonas áridas. Oficina Regional de la FAO para América Latina y el Caribe. 305 p. 


\section{Soberon, J.}

1998. Prologue. En: Cante, A. C.; Charles E. (Eds.). Threatened Cacti of Mexico. Identification Guide to Threatened Cacti of Mexico. Fideicomiso. Fondo para la Biodiversidad, México. D.F. 210 p.

Taiz, L.; Zeiger, E.

2006. Fisiología Vegetal. 13 ed. USA, Universitat Jaume. Castellón, España. 509 p.
Toogood, A.

2002. Propagating Plant a Darling Kinderley Book. Royal Horticulture Society. Barcelona, España. 192 p.

Vásquez E.;Valiente., A.; Briones., A., Bolongrado, C.; Ezcurra, E.; Rosas, M.; Núñez, H.; Barnard, G.

1995. Spatial relationships between cacti and nurse. In: a semiarid environment IN central México. Journal of Vegetation Science, 2: 15-20. 